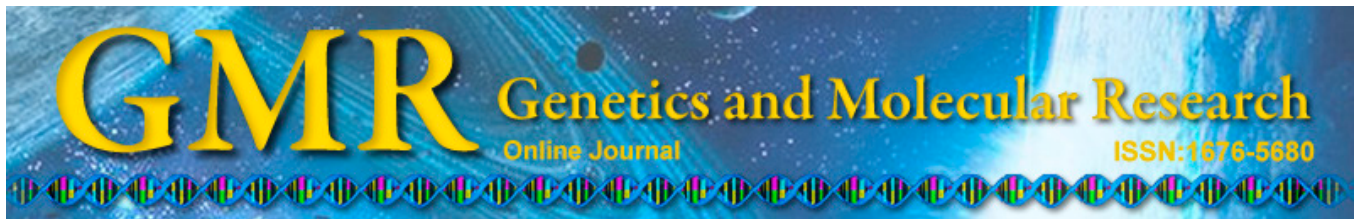

\title{
Identification of highly expressed host microRNAs that respond to white spot syndrome virus infection in the Pacific white shrimp Litopenaeus vannamei (Penaeidae)
}

\author{
D.G. Zeng*, X.L. Chen*, D.X. Xie, Y.Z. Zhao, Q. Yang, H. Wang, Y.M. Li \\ and X.H. Chen \\ Guangxi Key Laboratory of Aquatic Genetic Breeding and Healthy Aquaculture, \\ Guangxi Academy of Fisheries Sciences, Nanning, China \\ *These authors contributed equally to this study. \\ Corresponding author: X.H. Chen \\ E-mail: gxnnscs@126.com
}

Genet. Mol. Res. 14 (2): 4818-4828 (2015)

Received June 24, 2014

Accepted November 3, 2014

Published May 11, 2015

DOI http://dx.doi.org/10.4238/2015.May.11.14

\begin{abstract}
MicroRNAs (miRNAs) are known to play an important role in regulating both adaptive and innate immunity. Pacific white shrimp (Litopenaeus vannamei) is the most widely farmed crustacean species in the world. However, little is known about the role miRNAs play in shrimp immunity. To understand the impact of viral infection on miRNA expression in shrimp, we used high-throughput sequencing technology to sequence two small RNA libraries prepared from $L$. vannamei under normal and white spot syndrome virus (WSSV) challenged conditions. Approximately 19,312,189 and 39,763,551 raw reads corresponding to $17,414,787$ and $28,633,379$ high-quality mappable reads were obtained from the two libraries, respectively. Twelve conserved miRNAs and one novel miRNA that were highly expressed (>100 RPM) in L. vannamei were identified. Of the identified miRNAs, 8 were differentially expressed in response to the virus
\end{abstract}


infection, of which 1 was upregulated and 7 were downregulated. The prediction of miRNA targets showed that the target genes of the differentially expressed miRNAs were related to immunity, apoptosis, and development functions. Our study provides the first characterization of $L$. vannamei miRNAs in response to WSSV infection, which will help to reveal the roles of miRNAs in the antiviral mechanisms of shrimp.

Key words: MicroRNAs; Litopenaeus vannamei;

White spot syndrome virus; High-throughput sequencing

\section{INTRODUCTION}

Pacific white shrimp (Litopenaeus vannamei) is the most widely cultured crustacean species in the world due to its high yield and low-demand for concentration of salt (Zhou et al., 2012). Over the last three decades, viral pathogens, in particular white spot syndrome virus (WSSV), have seriously threatened the shrimp aquaculture industry and caused severe economic losses (Naylor et al., 2000). For sustainable shrimp farming, systematic approaches to prevent and/or control viral diseases of shrimp are of great significance. Therefore, studies of the immune system of shrimp, including the molecular responses and defense mechanisms in shrimp against viral pathogens, are particularly important.

MicroRNAs (miRNAs) are a class of approximately 22-nucleotide (nt) single strand, endogenous, non-coding RNAs that post-transcriptionally regulate gene expression by cleaving or inhibiting the translation of target gene transcripts in metazoan animals, plants, and protozoa (Bartel, 2004). It has been demonstrated that miRNAs play crucial roles in a large variety of biological and metabolic processes in cells, such as organ development, metabolism, signal transduction, cell proliferation, and biotic and abiotic stress responses (Xu et al., 2013). It is also becoming increasingly clear that miRNAs have important functions through binding to the 3'-untranslated region (UTR) of the target mRNA in regulating the innate and adaptive immune system (Ha, 2011). Thus, subsequent identification of the association between immune-related genes and their miRNAs is essential to better understand the immune defense mechanisms of animals.

Since miRNAs were first identified in Caenorhabditis elegans, thousands of miRNAs have been discovered from various organisms, including mammals, plants, insects, nematodes, and viruses (Griffiths-Jones et al., 2006). Several studies have reported identification of miRNAs in crustaceans, including Procambarus clarkii (Ou et al., 2013), Marsupenaeus japonicus (Huang et al., 2012), Eriocheir sinensis (Ou et al., 2012), and Parhyale hawaiensis (Blythe et al., 2012). Initially, miRNAs were identified using traditional Sanger sequencing techniques. Recently, high-throughput sequencing technologies, such as the Illumina/Solexa platform, Applied Biosystems Solid platform, and Roche pyrosequencing platform, have been successfully developed and widely applied for miRNA discovery in various organisms (Hallman et al., 2013; Severino et al., 2013). These high-throughput sequencing technologies provide a rapid and high-throughput method to identify miRNAs and their expression profile.

Despite the large number of miRNAs that have been identified and deposited in the miRBase, miRNAs in L. vannamei have not yet been discovered. In this study, we present the first high-throughput sequencing approach to identify and profile miRNA expression in WSSV-challenged $L$. vannamei, providing an important reference basis for further elucidating the role miRNAs play in regulating immune networks in this important crustacean species. 


\title{
MATERIAL AND METHODS
}

\begin{abstract}
Animals
L. vannamei from a specific pathogen-free (SPF) strain (National and Guangxi Shrimp Genetic Breeding Center, China) were used in this study. The shrimp (4-5 g in body weight) were maintained in flasks with $1000 \mathrm{~L}$ of seawater ( $32 \mathrm{ppt}$ salinity, 25 to $26^{\circ} \mathrm{C}$ ) and acclimatized for 3 days prior to the experiments.
\end{abstract}

\section{Preparation of viral inoculum}

The WSSV strain used to challenge the shrimp was isolated by our laboratory in 2008 from a $L$. vannamei shrimp in China. The WSSV suspension was prepared from frozen $\left(-80^{\circ} \mathrm{C}\right) \mathrm{WSSV}$-infected L. vannamei as described previously (James et al., 2010). The WSSV concentration was determined in our previous experiment, in which real-time quantitative PCR methods and reference samples containing gradient concentrations of WSSV were used (Zeng et al., 2013).

\section{Virus infection}

Ten shrimp in the challenge group were injected with $20 \mu \mathrm{L}$ WSSV suspension (diluted $1 \times 10^{6} \mathrm{~W} / \mathrm{v}$, approximately $1 \times 10^{5} \mathrm{WSSV}$ copies $/ \mathrm{g}$, a dose sufficient to cause $100 \%$ mortality in 5-7 days). In parallel, 10 shrimp in the control group were injected with SPF shrimp extract at an equivalent dilution. At $24 \mathrm{~h}$ after injection, the hepatopancreas tissues of each shrimp were collected and stored in liquid nitrogen until RNA isolation.

\section{Small RNA library construction and sequencing}

Total RNA was extracted from the shrimp hepatopancreas using Trizol reagent (Invitrogen, USA) following the manufacturer protocol, and the small RNA fractions, approximately 17-30 nt in length, were subsequently purified by electrophoretic separation on a $15 \%$ denaturing polyacrylamide gel. Equal amounts of the small RNA samples from each group were then pooled for cDNA synthesis and sequencing. The small RNA libraries were sequenced at the Beijing Autolab Biotechnology Company (China) using Solexa sequencing technology (Illumina).

\section{Identification of conserved and novel miRNAs in L. vannamei}

Raw sequencing reads were quality trimmed using the Illumina's software. After removing the adapter, contaminated sequences, and low quality sequences, clean sRNA reads of 17-26 nt were combined into unique sequences. Subsequently, the unique sequences were analyzed against mRNAs from crustacean species using BLAST, the Rfam database (http://rfam. sanger.ac.uk/), and Repbase (http://www.girinst.org/) to remove mRNA, snRNA, snoRNA, rRNA, tRNA, and repeat sequences. The high-quality unique sequences were then mapped to the Daphnia pulex genome (Daphnia_pulex.allmasked.gz) and the EST sequences of the shrimp L. vannamei using the SOAP software (http://soap.genomics.org.cn/; Luo et al., 2012). Perfect sRNA matches were retained for further analysis. The mapped sequences were aligned 
against known mature arthropod miRNAs in miRBase 18.0 (http://www.mirbase.org/; Kozomara and Griffiths-Jones, 2011), without allowing for mismatch. The Mfold program (http:// mfold.rna.albany.edu/; Zuker, 2003) was employed to identify characteristic stem-loop structures for miRNA precursors.

\section{Comparing the expression of miRNAs between the two libraries}

For differential miRNA expression analyzes, reads per million reads (RPM) were used as the value of normalized miRNA expression levels. Statistical comparison of RPM values between the uninfected and the WSSV-infected libraries was conducted using the web tool IDEG6 (http://telethon.bio.unipd.it/bioinfo/IDEG6_form/; Romualdi et al., 2003). A fold change greater than 2 was considered to be a significant difference. If the total RPM value of a miRNA was less than 100, it was removed before comparison due to low expression levels.

To validate miRNA expression, six differentially expressed miRNAs were randomly selected for stem-loop real-time RT-PCR analysis. RNAs were transcribed into cDNA using the PrimeScript miRNA cDNA Synthesis Kit (TaKaRa Bio Inc., Dalian, China) with stem-loop RT-primers, according to the manufacturer protocol. Thereafter, real-time quantitative PCR was performed with the SYBR Green PCR Master Mix Kit (TOYOBO, Japan) in a $20-\mu$ L reaction. The PCR mix included $10 \mu \mathrm{L} 2 X$ SYBR Green PCR Master Mix, $5 \mu \mathrm{L}$ cDNA for each miRNA (1:20 dilution), and $5 \mu \mathrm{M}$ of each forward and reverse primers. The $L$. vannamei $18 \mathrm{~S}$ rRNA gene was used as the endogenous control. The cycle conditions were as follows: $95^{\circ} \mathrm{C}$ for 10 min, followed by 45 cycles of $95^{\circ} \mathrm{C}$ for $15 \mathrm{~s}, 60^{\circ} \mathrm{C}$ for $34 \mathrm{~s}$, and $72^{\circ} \mathrm{C}$ for $30 \mathrm{~s}$. The primer pairs for $18 \mathrm{~S}$ rRNA gene were as follows: forward 5'-CGTCGCTACTACCGATTGAA TGGTC-3' and reverse 5'-TTCACCTACGGAAACCTTGTTACGACT-3'. The primers for miRNAs are listed in Table 1.

Table 1. Primers for stem-loop real-time RT-PCR analysis of miRNAs in Litopenaeus vannamei.

\begin{tabular}{|c|c|c|c|}
\hline miRNA name & RT-primer & Forward primer & Reverse primer \\
\hline dme-miR-991-5p & $\begin{array}{l}\text { GTCGTATCCAGTGCAGGGTCCGAGGT } \\
\text { ATTCGCACTGGATACGACATTAAT }\end{array}$ & GCGCTTCCCAACTACATCT & GTGCAGGGTCCGAGGT \\
\hline dme-miR-315-5p & $\begin{array}{l}\text { GTCGTATCCAGTGCAGGGTCCGAGGT } \\
\text { ATTCGCACTGGATACGACGCCTTC }\end{array}$ & GCGCTTTTGATTGTTGCTCA & GTGCAGGGTCCGAGGT \\
\hline dme-miR-133-3p & $\begin{array}{l}\text { GTCGTATCCAGTGCAGGGTCCGAGGT } \\
\text { ATTCGCACTGGATACGACACAGCT }\end{array}$ & GCGCTTGGTCCCCTTCAACC & GTGCAGGGTCCGAGGT \\
\hline dme-miR-184-3p & $\begin{array}{l}\text { TCCAGTGCAGGC } \\
\text { ACTGGATACGAC }\end{array}$ & GCGCTGGACGGAGAACTGA & GTGCAGGGTCCGAGGT \\
\hline dme-miR-1-3p & $\begin{array}{l}\text { GTCGTATCCAGTGCAGGGTCCGAGGT } \\
\text { ATTCGCACTGGATACGACCTCCAT }\end{array}$ & GCGCTGGAATGTAAAGAAGT & GTGCAGGGTCCGAGGT \\
\hline dme-let-7-5p & $\begin{array}{l}\text { GTCGTATCCAGTGCAGGGTCCGAGGT } \\
\text { ATTCGCACTGGATACGACACTATA }\end{array}$ & GCGCTGAGGTAGTAGGTTG & GTGCAGGGTCCGAGGT \\
\hline dme-bantam-3p & $\begin{array}{l}\text { GTCGTATCCAGTGCAGGGTCCGAGGT } \\
\text { ATTCGCACTGGATACGACAATCAG }\end{array}$ & GCGCTGAGATCATTGTGAAAG & GTGCAGGGTCCGAGGT \\
\hline dme-miR-2a-3p & $\begin{array}{l}\text { GTCGTATCCAGTGCAGGGTCCGAGGT } \\
\text { ATTCGCACTGGATACGACGCTCAT }\end{array}$ & GCGCTATCACAGCCAGCTTTG & GTGCAGGGTCCGAGGT \\
\hline dme-miR-276a-3p & $\begin{array}{l}\text { ICCGAGGT } \\
\text { GAGCA }\end{array}$ & GCGCTAGGAACTTCATACCG & GTGCAGGGTCCGAGGT \\
\hline dme-miR-10-5p & $\begin{array}{l}\text { GTCG } \\
\text { ATTC }\end{array}$ & GCGCTACCCTGTAGATCCGA & GTGCAGGGTCCGAGGT \\
\hline dme-n & $\begin{array}{l}\text { GTCGTATCCAGTGC } \\
\text { ATTCGCACTGGATAC }\end{array}$ & GCGCTAATACTGTCAGGTAAA & GTGCAGGGTCCGAGGT \\
\hline dme-miR-305-5p & $\begin{array}{l}\text { GTCGTATCCAGTGCAGGGTCCGAGGT } \\
\text { ATTCGCACTGGATACGACAGAGCA }\end{array}$ & GCGCATTGTACTTCATCAGG & GTGCAGGGTCCGAGGT \\
\hline lva-miR-100 & $\begin{array}{l}\text { GTCGTATCCAGTGCAGGGTCCGAGGT } \\
\text { ATTCGCACTGGATACGACCACAAG }\end{array}$ & GCGCAACCCGTAGATCCGAA & GTGCAGGGTCCGAGGT \\
\hline
\end{tabular}




\section{Prediction of miRNA targets}

The mRNA sequences of $L$. vannamei from the GenBank database were used to predict miRNA target genes. These mRNA sequences were assembled to non-redundant sequences comprising contigs and singlets using the iAssembler program (http://bioinfo.bti.cornell.edu/ tool/iAssembler; Zheng et al., 2011). Then, their corresponding 3'UTR sequences were determined using the UTRScan program (http://itbtools.ba.itb.cnr.it/utrscan; Grillo et al., 2010). A computational target prediction algorithm, RNAhybrid (http://bibiserv.techfak.uni-bielefeld. de/rnahybrid/) was used to predict the genes targeted by miRNAs (Kruger and Rehmsmeier, 2006). The functions of these targets were analyzed by Gene Ontology terms (GO) using the BLAST2GO program (http://www.BLAST2go.org/) with an E value threshold of less than $10^{-5}$.

\section{RESULTS}

\section{Solexa sequencing of small RNAs}

To identify miRNAs involved in the response of L. vannamei to WSSV infection, we created two small RNA libraries from pooled small RNAs extracted from the hepatopancreas of uninfected (control) and WSSV-infected shrimp. The two libraries were subjected to Solexa sequencing, generating approximately 19,312,189 (uninfected) and 39,763,551 (infected) raw reads, respectively. After the sequence processing steps of quality control and adapter removal, a total of 17,414,787 and 28,633,379 cleaned reads were harvested in the uninfected library and the infected library, respectively. After comparing the small RNA sequences with mRNAs from crustacean species, the Rfam database, and Repbase, the reads of degraded mRNA, snRNA, snoRNA, rRNA, tRNA, and repeat sequences in the libraries were removed. The remaining reads were retained for miRNA analysis. The length distribution analysis showed that approximately $70 \%$ of the small RNAs varied from 20-23 nt in length, with the 22 -nt class being the most abundant in total sequence number, followed by the 21-, 20-, and 23-nt classes (Figure 1).

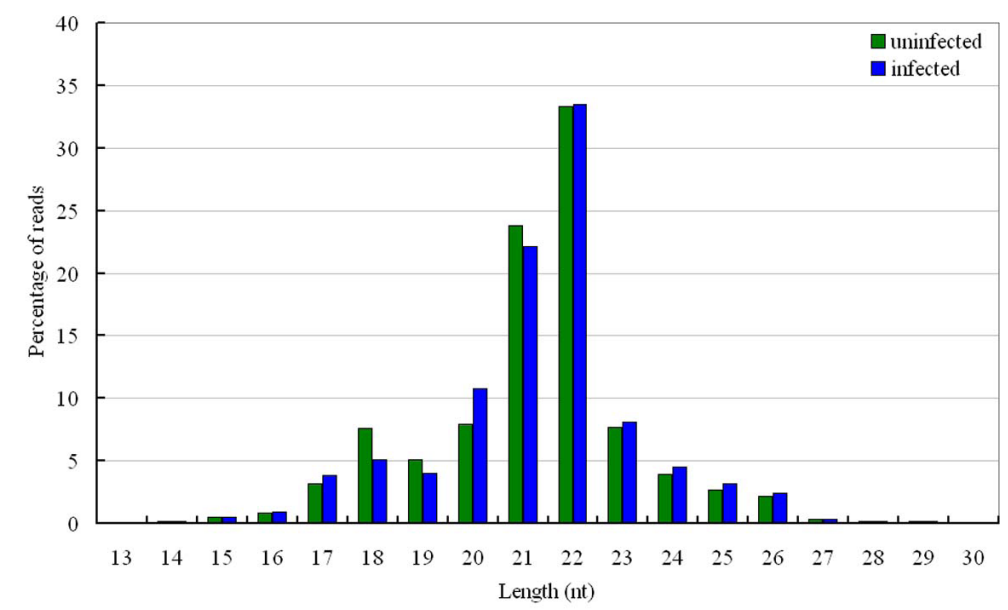

Figure 1. Length distribution of small RNAs in the uninfected and infected libraries from Litopenaeus vannamei. 


\section{Identification of miRNAs in L. vannamei}

Recently, it has been reported that miRNAs expressed below 100 RPM are unlikely to be functional miRNAs (Mullokandov et al., 2012). Using 100 RPM as a threshold for functional expression, we identified twelve conserved miRNAs in $L$. vannamei by comparing them with currently known arthropod mature miRNAs in miRBase and analyzing the hairpin structures for their precursors. In addition, we identified one putatively novel L. vannamei miRNA (named lva-miR-100 in this study), which showed no homology to known arthropod miRNAs in miRBase. The precursor of the lva-miR-100 miRNA had a typical stem-loop structure (Figure 2), and mature lva-miR-100 was highly expressed in both the uninfected and infected libraries. There was a large dynamic range in the expression of the L. vannamei miRNAs. Two miRNAs (lva-miR-100 and dme-bantam-3p) were expressed at very high levels $(>100,000$ reads), with the majority being expressed at relatively low levels (around tens of thousands of reads). Table 2 shows the miRNAs expressed in L. vannamei above 100 RPM.

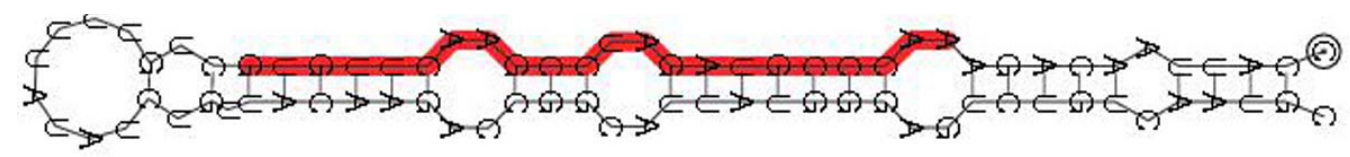

Figure 2. Predicted stem-loop structures of the precursor of lva-miR-100 (mature miRNA positions highlighted in red).

\begin{tabular}{|c|c|c|c|c|}
\hline miRNA name & Length (nt) & Sequence & Reads in uninfected & Reads in infected \\
\hline dme-miR-991-5p & 21 & UUCCCAACUACAUCUAUUAAU & 323 & 5,171 \\
\hline dme-miR-315-5p & 22 & UUUUGAUUGUUGCUCAGAAGGC & 1,972 & 630 \\
\hline dme-miR-133-3p & 22 & UUGGUCCCCUUCAACCAGCUGU & 36,353 & 34,747 \\
\hline dme-miR-184-3p & 21 & UGGACGGAGAACUGAUAAGGG & 50,106 & 44,460 \\
\hline dme-miR-1-3p & 22 & UGGAAUGUAAAGAAGUAUGGAG & 35,745 & 51,296 \\
\hline dme-let-7-5p & 21 & UGAGGUAGUAGGUUGUAUAGU & 51,403 & 29,734 \\
\hline dme-bantam-3p & 23 & UGAGAUCAUUGUGAAAGCUGAUU & 172,934 & 84,443 \\
\hline dme-miR-2a-3p & 23 & UAUCACAGCCAGCUUUGAUGAGC & 2,433 & 1,610 \\
\hline dme-miR-276a-3p & 22 & UAGGAACUUCAUACCGUGCUCU & 10,206 & 5,590 \\
\hline dme-miR-10-5p & 22 & UACCCUGUAGAUCCGAAUUUGU & 6,092 & 2,361 \\
\hline dme-miR-8-3p & 23 & UAAUACUGUCAGGUAAAGAUGUC & 2,566 & 2,498 \\
\hline dme-miR-305-5p & 22 & AUUGUACUUCAUCAGGUGCUCU & 13,517 & 9,713 \\
\hline lva-miR-100 & 22 & AACCCGUAGAUCCGAACUUGUG & 338,494 & 380,972 \\
\hline
\end{tabular}

\section{Differentially expressed miRNAs and prediction of potential target genes}

To identify miRNAs involved in virus infection, the normalized expressions of conserved miRNAs in the uninfected and infected small RNA libraries were compared. The miRNAs with changes in expression levels greater than 2-fold are presented in Table 3 . To verify the existence and expression change of the differentially expressed miRNAs, the same RNA preparation used in the Illumina/Solexa sequencing was subjected to stem-loop qPCR analysis. The results show that the qRT-PCR analysis agreed well with the Illumina/Solexa sequencing analysis. The fold change of the differentially expressed miRNAs between the uninfected and infected small RNA libraries are also presented in Table 3.

In total, eight differentially expressed miRNAs were identified between the uninfected and infected small RNA libraries. Of these eight differentially expressed miRNAs, one was 
significantly upregulated while seven were significantly downregulated upon challenge with WSSV. The results indicate that WSSV infection has a significant impact on several miRNAs in L. vannamei.

Identifying target genes of miRNAs is important to understand their specific biological functions. To predict the target genes of the differentially expressed miRNAs, a total of 162,933 EST sequences of L. vannamei from the GenBank EST database were assembled to 31,845 non-redundant sequences comprising contigs and singlets. Subsequently, their corresponding 3'UTR sequences were determined and were subjected to prediction for miRNA target genes. The highest scoring target genes predicted by RNAhybrid algorithm for each miRNA are shown in Table 3. The results of GO terms analysis indicated that these target genes were mainly related to immunity (lysozyme, Toll 2, and C-type lectin), apoptosis (eukaryotic initiation factor 4A and inositol-requiring enzyme-1), and development (activating transcription factor 4, hemolymph clottable protein, and tumor necrosis factor receptor-associated factor 6) functions. Figure 3 shows the miRNA target sites predicted by the RNAhybrid.

\begin{tabular}{|c|c|c|c|c|c|}
\hline miRNA name & $\begin{array}{l}\text { RPM in } \\
\text { uninfected }\end{array}$ & $\begin{array}{l}\text { RPM in } \\
\text { infected }\end{array}$ & $\begin{array}{l}\text { Infected/uninfected } \\
\text { (Solexa) }\end{array}$ & $\begin{array}{l}\text { Infected/uninfected } \\
\text { (qPCR) }\end{array}$ & $\begin{array}{l}\text { Highest scoring target } \\
\text { gene in } L \text {. vannamei }\end{array}$ \\
\hline dme-miR-991-5p & 18.55 & 180.59 & 9.74 & $10.63 \pm 0.64$ & activating transcription factor 4 \\
\hline dme-miR-315-5p & 113.24 & 22.00 & 0.19 & $0.18 \pm 0.01$ & C-type lectin \\
\hline dme-let-7-5p & 2951.69 & 1038.44 & 0.35 & $0.41 \pm 0.04$ & Toll 2 \\
\hline dme-bantam-3p & 9930.30 & 2949.11 & 0.30 & $0.28 \pm 0.01$ & Inositol-requiring enzyme-1 \\
\hline dme-miR-2a-3p & 139.71 & 56.23 & 0.40 & $0.38 \pm 0.02$ & Eukaryotic initiation factor $4 \mathrm{~A}$ \\
\hline dme-miR-276a-3p & 586.05 & 195.23 & 0.33 & $0.30 \pm 0.02$ & $\begin{array}{l}\text { Tumor necrosis factor receptor- } \\
\text { Associated factor } 6\end{array}$ \\
\hline dme-miR-10-5p & 349.82 & 82.46 & 0.24 & $0.23 \pm 0.01$ & Hemolymph clottable protein \\
\hline dme-miR-305-5p & 776.18 & 339.22 & 0.44 & $0.47 \pm 0.09$ & Invertebrate-type lysozyme \\
\hline
\end{tabular}

A

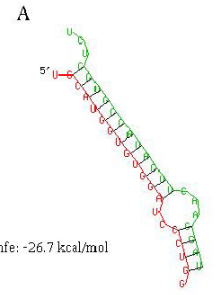

$\mathrm{E}$

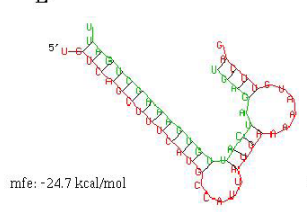

B
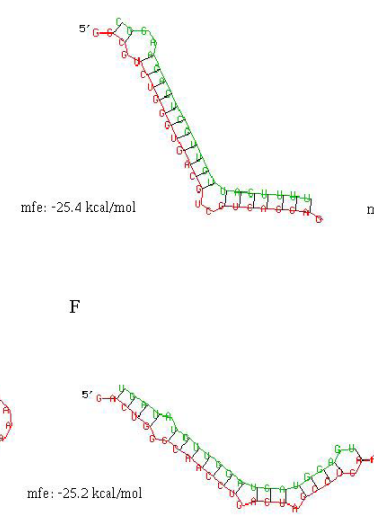
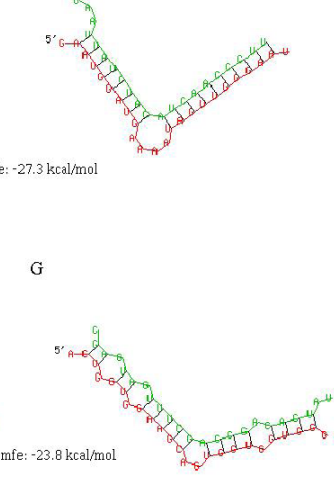

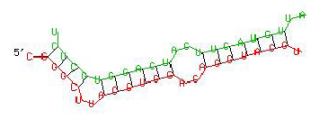

mfe : $-25.0 \mathrm{kcca} / \mathrm{mol}$

Figure 3. Prediction of miRNA binding sites within the Litopenaeus vannamei target mRNA sequences at the indicated 3'UTR nucleotide positions using RNAhybrid. The letters represent miRNAs as follows: A: dme-miR276a-3p; B: dme-miR-315-5p; C: dme-miR-991-5p; D: dme-miR-305-5; E: dme-bantam-3p; F: dme-let-7-5p; G: dme-miR-2a-3p; H: dme-miR-10-5p. 


\section{DISCUSSION}

Recent studies have shown that miRNAs play important roles in the regulation of the innate and adaptive immune system in animals, plants, and protozoa (Carrington and Ambros, 2003; Davidson-Moncada et al., 2010; O'Connell et al., 2010). Identifying miRNAs and understanding their roles in host-pathogen interactions is becoming increasingly important for studying the host's immune system, disease control, and breeding for disease resistance. Although several miRNAs have been identified through computational or experimental approaches in crustaceans, there is no sequence or functional information available for miRNAs in L. vannamei, which is economically important in the shrimp aquaculture industry.

In this study, we used the Illumina/Solexa technology for deep sequencing of small RNAs to identify miRNAs in L. vannamei. The length distribution pattern obtained indicates that the majority of small RNAs from the libraries were 20-24 nt in length, which is typical for products processed by the enzyme Dicer. This distribution pattern is similar to those observed in previous reports of crustacean small RNA sequencing using Illumina/Solexa technology. We also compared our small RNA libraries from L. vannamei against known miRNAs from the miRBase database to identify miRNAs in L. vannamei. Since miRNAs expressed below 100 RPM are not considered to be functional miRNAs, we only identified the highly expressed miRNAs (RPM >100). In total, 13 miRNAs, including 12 conserved miRNAs and one novel miRNA, were identified in L. vannamei. Interestingly, the most highly expressed miRNA, lvamiR-100, exhibited a particularly high expression level. lva-miR-100 belongs to the miR-99 family, and it has been demonstrated that miR-99 family members regulate cell proliferation, cell migration, and AKT/m TOR signaling (Jin et al., 2013; Li et al., 2013). Considering the particularly high expression of lva-miR-100 in L. vannamei, we suggest that miR-99 family members may have important biological functions in shrimp. However, there was no significant difference in lva-miR-100 expression between the uninfected and infected libraries, indicating that miR-99 family members may not be significantly affected by viral infection.

As it is well known, miRNAs are important regulators of many cellular processes, such as apoptosis, differentiation, metabolism, signal transduction, and stress responses (Bartel, 2004; Krutzfeldt and Stoffel, 2006; Hime and Somers, 2009; Fernandez-Hernando et al., 2011). Virus infection can disturb and subvert host cellular processes and functions by affecting cellular miRNAs (Scaria et al., 2006; Ghosh et al., 2009; Skalsky and Cullen, 2010). miRNA profiling is therefore necessary to understand virus-host interactions. In this study, $L$. vanname $i$ was challenged with WSSV to characterize the host miRNAs involved in the response to viral infection. The comparative analysis of miRNA transcript changes between uninfected and infected libraries revealed eight differentially expressed miRNAs, of which one miRNA was upregulated and seven were downregulated. These host miRNAs are involved in virus-host interactions, most of which are related to host immune responses. Among the differentially expressed miRNAs, the most highly expressed miRNA is dme-bantam-3p, which belongs to the bantam family, a family of miRNAs that has been shown to affect tissue growth by stimulating cell proliferation and inhibiting cell apoptosis in Drosophila (Thompson and Cohen, 2006; Jaklevic et al., 2008). In the present study, dme-bantam-3p was expressed 0.30fold greater in the infected library than in the uninfected library, indicating that WSSV may stimulate apoptosis of host cells by inhibiting the expression of bantam family members. The second most highly expressed miRNA, dme-let-7-5p, was also significantly downregulated $(0.35$-fold $)$ in the infected library compared to the uninfected library. The dme-let-7-5p miR- 
NA belongs to the let-7 family, a family of miRNAs that has several well-documented roles in immunity. It has been previously reported that let-7 family members in mammals can regulate expression of major cytokine-inducible proteins in the immune response against pathogen infection (Hu et al., 2009). For example, several let-7 family members, including let-7a, were shown to be downregulated in murine macrophages during Salmonella infection (Schulte et al., 2011). The results suggest that the let-7 miRNAs play critical roles in shrimp immune response against viral infection.

Identifying target genes regulated by miRNAs is essential for studying their specific biological functions (Bentwich, 2005). In order to understand the biological functions of the differentially expressed miRNAs identified in this study, we predicted their target genes using bioinformatic methods. Currently, the most efficient tool available for identifying target genes is the bioinformatic approach, and many target prediction programs have been developed and used for the newly identified miRNAs, including RNAhybrid, PicTar, TargetScan, miRanda, miRbase-Targets, and MicroInspector, etc. (Thomas et al., 2010; Lindow, 2011). In this study, we predicted miRNA targets in L. vannamei using RNAhybrid, a program that can predict multiple potential binding sites of miRNAs in 3'UTRs. The results showed that most of the targets were related to immune responses, gene expression regulation, signal transduction, and metabolism, indicating that the corresponding miRNAs are involved in various processes of shrimp defense against viruses. miRNAs usually downregulate target genes by binding to the complementary sites in the 3'UTR of the targets, and variations in miRNA patterns against virus infection can lead to differences in gene expression. Accordingly, miRNA expression profiling may be used to evaluate shrimp immunity.

In summary, we employed the Illumina/Solexa sequencing technique to investigate the miRNA profile of $L$. vannamei challenged with WSSV. Our study provides the first characterization of $L$. vannamei miRNAs in response to virus infection. The results showed that a total of thirteen highly expressed miRNAs in L. vannamei were obtained, eight of which were differentially expressed in response to WSSV infection. Although the molecular functions of these miRNAs and their associated target genes remain largely unknown, this study provides valuable information on the antiviral mechanism in shrimp and the role of the differentially expressed miRNAs in response to virus infection. Furthermore, the large number of small RNAs obtained in this study provides a strong basis for future genomic research on shrimp.

\section{ACKNOWLEDGMENTS}

Research supported by the National High Technology Research and Development Program of China (Grant \#2012AA10A404) and the National Natural Science Foundation of China (\#31360643).

\section{REFERENCES}

Bartel DP (2004). MicroRNAs: genomics, biogenesis, mechanism, and function. Cell 116: 281-297.

Bentwich I (2005). Prediction and validation of microRNAs and their targets. FEBS Lett. 579: 5904-5910.

Blythe MJ, Malla S, Everall R, Shih YH, et al. (2012). High through-put sequencing of the Parhyale hawaiensis mRNAs and microRNAs to aid comparative developmental studies. PLoS One 7: e33784.

Carrington JC and Ambros V (2003). Role of microRNAs in plant and animal development. Science 301: 336-338.

Davidson-Moncada J, Papavasiliou FN and Tam W (2010). MicroRNAs of the immune system: roles in inflammation and cancer. Ann. NY Acad. Sci. 1183: 183-194. 
Fernandez-Hernando C, Suarez Y, Rayner KJ and Moore KJ (2011). MicroRNAs in lipid metabolism. Curr. Opin. Lipidol. 22: 86-92.

Ghosh Z, Mallick B and Chakrabarti J (2009). Cellular versus viral microRNAs in host-virus interaction. Nucleic Acids Res. 37: 1035-1048.

Griffiths-Jones S, Grocock RJ, van Dongen S, Bateman A, et al. (2006). miRBase: microRNA sequences, targets and gene nomenclature. Nucleic Acids Res. 34: D140-144.

Grillo G, Turi A, Licciulli F, Mignone F, et al. (2010). UTRdb and UTRsite (RELEASE 2010): a collection of sequences and regulatory motifs of the untranslated regions of eukaryotic mRNAs. Nucleic Acids Res. 38: D75-80.

Ha TY (2011). The Role of MicroRNAs in regulatory T cells and in the immune response. Immune Netw. 11: 11-41.

Hallman J, Avesson L, Reimegard J, Kaller M, et al. (2013). Identification and verification of microRNAs by highthroughput sequencing. Methods Mol. Biol. 983: 125-138.

Hime GR and Somers WG (2009). Micro-RNA mediated regulation of proliferation, self-renewal and differentiation of mammalian stem cells. Cell Adh. Migr. 3: 425-432.

Hu G, Zhou R, Liu J, Gong AY, et al. (2009). MicroRNA-98 and let-7 confer cholangiocyte expression of cytokineinducible Src homology 2-containing protein in response to microbial challenge. J. Immunol. 183: 1617-1624.

Huang T, Xu D and Zhang X (2012). Characterization of host microRNAs that respond to DNA virus infection in a crustacean. BMC Genomics 13: 159.

Jaklevic B, Uyetake L, Wichmann A, Bilak A, et al. (2008). Modulation of ionizing radiation-induced apoptosis by bantam microRNA in Drosophila. Dev. Biol. 320: 122-130.

James R, Thampuran N, Lalitha KV, Rajan LA, et al. (2010). Differential gene expression profile of the hepatopancreas of white spot syndrome virus infected Fenneropenaeus indicus by suppression subtractive hybridization. Fish Shellfish Immunol. 29: 884-889.

Jin Y, Tymen SD, Chen D, Fang ZJ, et al. (2013). MicroRNA-99 family targets AKT/mTOR signaling pathway in dermal wound healing. PLoS One 8: e64434.

Kozomara A and Griffiths-Jones S (2011). miRBase: integrating microRNA annotation and deep-sequencing data. Nucleic Acids Res. 39: D152-157.

Kruger J and Rehmsmeier M (2006). RNAhybrid: microRNA target prediction easy, fast and flexible. Nucleic Acids Res. 34: W451-454.

Krutzfeldt J and Stoffel M (2006). MicroRNAs: a new class of regulatory genes affecting metabolism. Cell Metab. 4: 9-12.

Li XJ, Luo XQ, Han BW, Duan FT, et al. (2013). MicroRNA-100/99a, deregulated in acute lymphoblastic leukaemia, suppress proliferation and promote apoptosis by regulating the FKBP51 and IGF1R/mTOR signalling pathways. $B r$. J. Cancer 109: 2189-2198.

Lindow M (2011). Prediction of targets for microRNAs. Methods Mol. Biol. 703: 311-317.

Luo R, Liu B, Xie Y, Li Z, et al. (2012). SOAPdenovo2: an empirically improved memory-efficient short-read de novo assembler. Gigascience 1: 18.

Mullokandov G, Baccarini A, Ruzo A, Jayaprakash AD, et al. (2012). High-throughput assessment of microRNA activity and function using microRNA sensor and decoy libraries. Nat. Methods 9: 840-846.

Naylor RL, Goldburg RJ, Primavera JH, Kautsky N, et al. (2000). Effect of aquaculture on world fish supplies. Nature 405: 1017-1024.

O'Connell RM, Rao DS, Chaudhuri AA and Baltimore D (2010). Physiological and pathological roles for microRNAs in the immune system. Nat. Rev. Immunol. 10: 111-122.

Ou J, Meng Q, Li Y, Xiu Y, et al. (2012). Identification and comparative analysis of the Eriocheir sinensis microRNA transcriptome response to Spiroplasma eriocheiris infection using a deep sequencing approach. Fish Shellfish Immunol. 32: 345-352.

Ou J, Li Y, Ding Z, Xiu Y, et al. (2013). Transcriptome-wide identification and characterization of the Procambarus clarkii microRNAs potentially related to immunity against Spiroplasma eriocheiris infection. Fish Shellfish Immunol. 35: 607-617.

Romualdi C, Bortoluzzi S, D’Alessi F and Danieli GA (2003). IDEG6: a web tool for detection of differentially expressed genes in multiple tag sampling experiments. Physiol. Genomics 12: 159-162.

Scaria V, Hariharan M, Maiti S, Pillai B, et al. (2006). Host-virus interaction: a new role for microRNAs. Retrovirology 3: 68.

Schulte LN, Eulalio A, Mollenkopf HJ, Reinhardt R. et al. (2011). Analysis of the host microRNA response to Salmonella uncovers the control of major cytokines by the let-7 family. EMBO J. 30: 1977-1989.

Severino P, Oliveira LS, Torres N, Andreghetto FM, et al. (2013). High-throughput sequencing of small RNA transcriptomes reveals critical biological features targeted by microRNAs in cell models used for squamous cell cancer research. BMC Genomics 14: 735.

Skalsky RL and Cullen BR (2010). Viruses, microRNAs, and host interactions. Annu. Rev. Microbiol. 64: 123-141.

Genetics and Molecular Research 14 (2): 4818-4828 (2015) 
Thomas M, Lieberman J and Lal A (2010). Desperately seeking microRNA targets. Nat. Struct. Mol. Biol. 17: 1169-1174. Thompson BJ and Cohen SM (2006). The Hippo pathway regulates the bantam microRNA to control cell proliferation and apoptosis in Drosophila. Cell 126: 767-774.

Xu L, Wang Y, Xu Y, Wang L, et al. (2013). Identification and characterization of novel and conserved microRNAs in radish (Raphanus sativus L.) using high-throughput sequencing. Plant Sci. 201-202: 108-114.

Zeng D, Chen X, Xie D, Zhao Y, et al. (2013). Transcriptome analysis of Pacific white shrimp (Litopenaeus vannamei) hepatopancreas in response to Taura syndrome Virus (TSV) experimental infection. PLoS One 8: e57515.

Zheng Y, Zhao L, Gao J and Fei Z (2011). iAssembler: a package for de novo assembly of Roche-454/Sanger transcriptome sequences. BMC Bioinformatics 12: 453.

Zhou J, Fang W, Yang X, Zhou S, et al. (2012). A nonluminescent and highly virulent Vibrio harveyi strain is associated with "bacterial white tail disease" of Litopenaeus vannamei shrimp. PLoS One 7: e29961.

Zuker M (2003). Mfold web server for nucleic acid folding and hybridization prediction. Nucleic Acids Res. 31: 34063415. 